\title{
Guided Sitting and Walking Meditations on Emotion
}

\author{
Ajahn Amaro ${ }^{1}$ \\ Published online: 8 April 2019 \\ (C) Amaravati Publications 2019
}

\section{Sitting Meditation}

Take a moment to notice how you feel, the effects of your day so far: what you thought, what you felt, what you have heard and seen, the feelings of the body.

Take a moment to simply attend, to look at them, to feel and notice them.

Are you excited? Bored? Warm? Cool? Depressed? Inspired? Full of mixed feelings? Whatever it might be, notice that right now it's like this. The body feels like this. The mind feels like this.

Then let the body settle and the spine straighten. Invite the body to sit in an energized, upright way. Allow the spine to grow to its full natural extension.

Let the body settle and then center the attention upon the breathing.

Allow the attention to settle on the feeling of the breath. Use that rhythm, that pattern of feeling, as a center point, a focal point for attention. When the mind wanders, follow it back; come back to the central point, the simplicity of this moment.

When you feel the mind is settled and there's a quality of poise and some ease, deliberately bring to mind a memory of some encounter, some occasion when you were blamed, threatened, and misunderstood. You can choose your own least favorite encounter if you wish. Bring to mind the memory of an occasion that has that type of emotional charge to it. Try not to go into a big story, but just bring the attention back to that event, that exchange, that engagement. Bring it to

Excerpted from I'm right, you're wrong! by Ajahn Amaro. Amaravati Publications, 2016.

Ajahn Amaro

ajahn.amaro@gmail.com

1 Amaravati Buddhist Monastery, Hemel Hempstead, Hertfordshire HP1 3BZ, UK mind. Let that emotion be triggered, fully born into your consciousness.

As the memory of that emotion, when you were attacked, threatened or criticized is triggered, see if you can find it in the body. What's the physical counterpart to that emotion? Where do you feel that?

If you are able to find such a feeling, such a sensation in the belly, the throat, the shoulders, wherever it might be, bring the attention to that feeling. Let the attention settle there, hold that feeling as fully as you can in awareness. Do not try to get rid of it, change it, buy into it, or push it away. Know the feelingthat it's like this.

As you let the feeling be fully known, bringing awareness to it, then with the rhythm of the breath, let yourself relax your attitude toward that feeling, let go of it, particularly using the out-breath to help carry it away. So the feeling is naturally eroding, it's washing through you, not by trying to "get rid of" it, but just like the waves of the sea slowly washing away a sandcastle, lapping in with each breath, washing around that feeling and slowly carrying it away, back to the ocean.

Let the attention rest with that feeling in the body. If the mind gets lost in stories, replaying dialogs, getting verbal about all the "this and that's", again let the out-breath carry that away and come back to that feeling, that sensation: the tightness in the belly, the tension in the shoulder, wherever you might feel it. Very consciously leave the words aside. Keep the attention with the simplicity, the directness of the physical feeling; stay with that, being open, patient, relaxed with it, letting it slowly, gently fade away, however long it takes. Stay with it.

Keep letting the out-breath have its effects, gently, steadily supporting the quality of release, relinquishment, relaxation. Stay with this until the body is fully relaxed, until you have completely let go of that sensation, that emotion, its effects washed utterly away. Once it's gone, sustain the attention on that absence. How does it feel with that tension gone, the body in a state of ease, relaxation?

We've watched that mood, that emotion born from nothing, born from the arousal of a memory, burst into being, rise up, 
flower and fade away; the flowers bloom and fade, the fruits fall, the leaves drop, sink back into the earth, and then it's all gone. It comes out of nothing and returns to nothing.

We've watched that whole cycle of experience come into being, do its thing, and fade away. And, throughout the whole process, there's been this quality of knowing, caring, careful attention, and noticing, appreciating this quality of spaciousness.

In the open space of the mind, we can once again invite that same cycle of feeling, bring up another occasion or the same one, the same memory of when we were threatened or hurt. Again, keep it as non-conceptual, as nonverbal as possible. Just remember: "That day, that letter, that conversation"; you do not need any more of a trigger than that. You know all the details already. And then let the whole process run again, bringing that to mind and immediately going to the feeling in the body: fear, selfdefensiveness, insecurity, whatever it might be. Notice where you feel it. Train the mind to stay with it in its simplicity. Feel it, know it, and be with it throughout its cycle.

Sometimes the cycle of feeling can go through its turnings very swiftly. A thought gets triggered and there's a flush of emotion:

"They all hate me!" And then there's recognition:

"Stop! Look! Feel that one!"

And in a couple of breaths it's gone. Completely finished.

See how this pattern works, become familiar with it, and learn the skill of non-entanglement, not clinging, letting go, releasing.

\section{Walking Meditation}

You can use walking meditation to explore the same area of reactivity, but aim to keep the feeling of the feet as the primary focal point - the rhythm of the feet touching the ground as you walk. I would also encourage not walking at a particularly slow pace. Walk as if on an ordinary gentle stroll, using the feeling of the feet touching the ground as a focal point for attention. When you notice the mind has picked up some kind of emotional reaction or thought, bring attention to the body and again let yourself feel it, know it, and relax with it. Do that for the first few minutes, maybe the first five or ten minutes or so, to settle into the walking practice. Then continue to develop this body awareness and the way that emotional states sit in the body.

After you have done that for five or ten minutes, use the same kind of theme as you did during the sitting meditation. Take some particular memory or attitude, say: "They all hate me!"- "they", the mysterious, undefined "they" who have such critical attitudes about our lives - take up that sense of emotional threat, of being misunderstood, by consciously bringing a particular incident or habitual attitude that you have to mind, and as you walk, notice how it sits in the body in a mobile state as you are moving and engaged in physical activity.

Again, see if you can attend to that, be aware of it and then let it go as you walk along. Sometimes the action of walking is like stirring the pot, so it makes the non-entanglement a little harder to realize, but it's a good exercise to see if you can relax into the walking and let go of the state, rather than letting the action of walking crank things up and inflate the negative reaction.

Publisher's Note Springer Nature remains neutral with regard to jurisdictional claims in published maps and institutional affiliations. 